\title{
Microstructural Analysis of Si Frameworks Induced by Electrochemical (De)Alloying Process
}

Dong-Su Ko ${ }^{1}$, Ken Ogata ${ }^{2}$, Sungho Jeon ${ }^{3}$, Changhoon Jung ${ }^{1}$, Junho Lee ${ }^{1}$, Soohwan Sul ${ }^{1}$, Hee-Goo Kim ${ }^{1}$, and Jai Kwang Shin ${ }^{1}$

1. Platform Technology Lab, Samsung Advanced Institute of Technology, Suwon, Republic of Korea

2. Samsung Research Institute of Japan, Mino, Japan

3. Energy Lab, Samsung Advanced Institute of Technology, Suwon, Republic of Korea

Porous silicon has attracted enormous attention for their landmark applications such as light emitting sources, sensors, actuators, drug delivery systems, and energy storage systems. In particular, a variety of selective etching techniques have been widely used to form unique porous structures of silicon [1]. However, it inevitably accompanies with gravimetric loss. Here, we demonstrate a new technique to form porous silicon, which is spontaneously formed with atomistic re-arrangement of silicon without gravimetric loss via single electrochemical (de)alloying with lithium. TEM reveals the key prerequisite to the formation of the unique porous structures.

Two different types of Si particles were used in this study to investigate the effect of Si crystallinity on the porous structures (highly defective poly-crystalline Si and single-crystalline Si with little defects). A Si framework was formed by electrochemical (de)alloying by lithium. Microstructural analysis was carried out using by TEM and tomography accompanying with chemical analysis conducted by EELS spectrum imaging.

Figure 1(a,c) shows TEM images of as-is poly-crystalline Si particles with defects such as grainboundary, twin-boundary, and stacking faults. After alloying and dealloying with lithium (1 cycle), Si microstructure was changed from crystalline to amorphous with framework structure. The framework structure typically consists of sub $2 \mathrm{~nm}$-width wires and ca. 3-4 nm pores, maintaining the original spherical shape. The bulk stripes $(\sim 10 \mathrm{~nm})$ in the framework structures consisted of only Si (Fig. 1(e)), which are probably derived from amorphized twins and stacking faults. They have more difficulties in being fully alloyed due to a higher energy cost to break Si-Si bonding. Unlike poly-crystalline, most of single-crystalline $\mathrm{Si}$ particles changed to amorphous $\mathrm{Si}$ without framework formation after 1 cycle. Some crystalline Si particles showed a similar microstructure to poly-crystalline Si particle after one electrochemical cycling. This is due to the existence of its stacking faults and twin defects in some crystalline Si particles. Without bulk stripes the framework structure developed after 4 complete cycles. Figure 2 shows the evolution of the framework structure by repeating (de)alloying. These unique 3dimentional microstructures were confirmed by tomography.

We can successfully form continuous $\mathrm{Si}$ frameworks with tunable size of pores by controlling the conditions of dealloying process. In our previous study [2], we showed that the unique dealloying dynamics is closely related to the formation of crystalline $\mathrm{Li}_{3.75} \mathrm{Si}$ phase during (de)alloying process. During dealloying process from crystalline $\mathrm{Li}_{3.75} \mathrm{Si}$ to amorphous $\mathrm{Li}_{\mathrm{x}} \mathrm{Si}$, the difference of $\mathrm{Li}$ diffusivity between crystalline $\mathrm{Li}_{3.75} \mathrm{Si}$ and amorphous $\mathrm{Li}_{\mathrm{x}} \mathrm{Si}$ phases may trigger Kirkendal voiding, which lead the formation of nano-pores. Moreover, the difference of ductility more easily induces nano-pores under tensile stress [3]. 


\section{References:}

[1] J. Erlebacher, M. J. Aziz, A.Karma, N. Dimitrov and K. Sieradzki, Nature 410 (2001), 450-453

[2] K. Ogata, S. Jeon, D.-S. Ko and et al, Nature Communication accepted (2018)

[3] H. Wang, X. Wang, S. Xia and H. B. Chew, The Journal of Chemical Physics 143 (2015), 104703
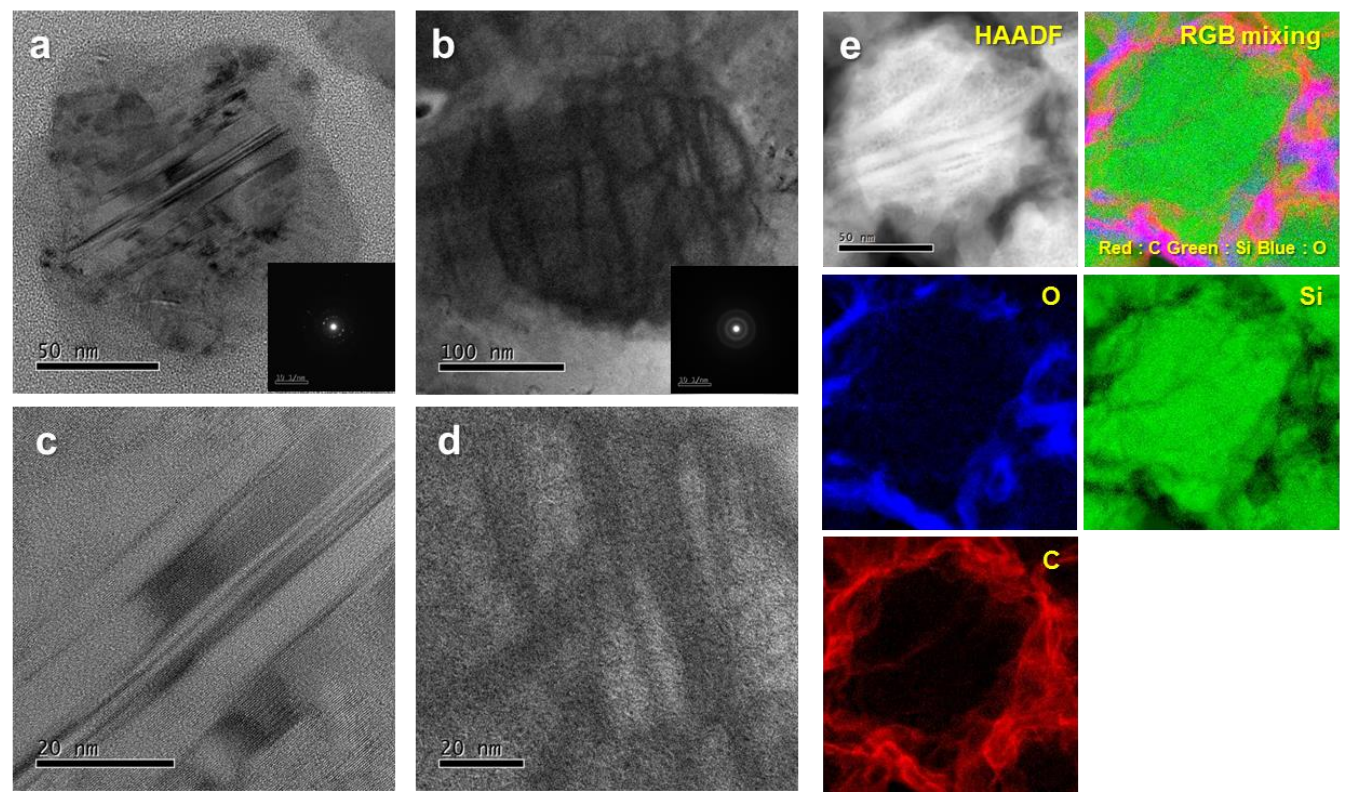

Figure 1. TEM images of (a, c) as-is poly-crystalline $\mathrm{Si}$ particle and (b, d) after 1 cycle (electrochemical lithiation and delithiation). (e) EELS SI mapping images of 1 cycled poly-crystalline Si particle.

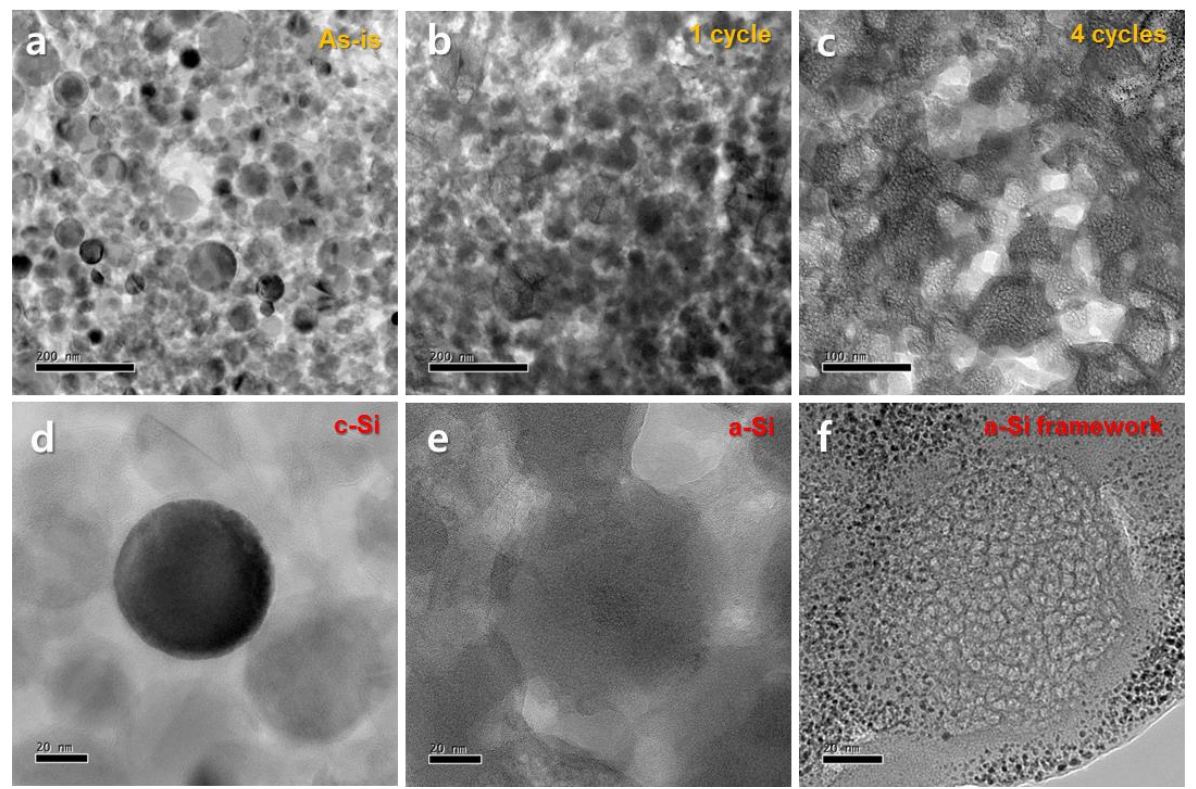

Figure 2. TEM images of (a, d) as-is single-crystalline Si particles, (b, e) after 1 cycle and (c, f) 4 cycles. 